Conclusions The increase in time to first severe exacerbation and first episode of asthma worsening found with the addition of tiotropium was not limited to specific subgroups of patients, including some characteristics that are usually found in patients with chronic obstructive pulmonary disease, such as former smoking, non-allergic status or minimal reversibility. Tiotropium seems effective across a broad spectrum of patients with severe persistent asthma who remain symptomatic and experience exacerbations despite the combination use of moderate- to high-dose inhaled corticosteroids plus long-acting beta agonists.

\section{P164 USE OF BETA-AGONISTS PRIOR TO HOSPITAL ATTENDANCE FOR SEVERE EXACERBATIONS OF ASTHMA: INSIGHTS FROM A RANDOMISED CONTROLLED TRIAL USING ELECTRONIC MONITORING OF INHALER USE}

${ }^{1} \mathrm{M}$ Patel, ${ }^{2} \mathrm{~J}$ Pilcher, ${ }^{1} \mathrm{D}$ Shaw, ${ }^{3} \mathrm{M}$ Weatherall, ${ }^{1} \mathrm{~A}$ Knox, ${ }^{2} \mathrm{R}$ Beasley; ${ }^{1}$ Division of Respiratory Medicine, University of Nottingham, Nottingham, UK; ${ }^{2}$ Medical Research Institute of New Zealand, Wellington, New Zealand; ${ }^{3}$ University of Otago, Wellington, New Zealand

\subsection{6/thoraxjnl-2013-204457.315}

Introduction Observational studies have reported that the overuse of inhaled beta-agonists during severe asthma is a common feature associated with a fatal outcome. However, patterns of actual use of beta-agonists prior to hospital attendance for severe exacerbations are poorly understood.

Objectives We have recently reported that in 303 adult asthma patients randomised to receive either combination budesonide/formoterol metered dose inhaler (MDI) as part of a single maintenance and reliever therapy regimen ('SMART') or as fixed-dose maintenance treatment with salbutamol MDI for relief ('Standard'), overuse of beta-agonists without subsequent medical review occurred commonly in both groups. We now report on the use of beta-agonists by patients who attended hospital with a severe exacerbation of asthma. Our hypothesis was that extremely high beta-agonist doses would be used by patients in both groups and that inhaled corticosteroid (ICS) nonadherence may occur in the Standard group during severe asthma.

Methods Data on MDI use, as measured by electronic monitoring, were extracted for each patient for the 14 24-hour periods before the attendance time at hospital for a severe exacerbation. Results Electronic data were available for $7 / 7$ and 9/11 hospital attendances in the SMART and Standard groups respectively. The median (range) daily number of actuations 14 days before hospital attendance was 4 (2 to 12) budesonide/formoterol in SMART and 4 (0 to 26 ) salbutamol and 2 ( 0 to 8 ) budesonide/formoterol in Standard. This increased to 11 (6 to 39) budesonide/formoterol in SMART and 25 ( 3 to 86 ) salbutamol and 4 (0 to 39 ) budesonide/formoterol in Standard, in the 24-hours before attendance. The median (range) maximum daily number of actuations was 14 (9 to 63) budesonide/formoterol in SMART and 46 (6 to 95) salbutamol in Standard. Repeated days of no ICS use occurred in 3/9 patients in the Standard group, despite concomitant salbutamol overuse.

Conclusions Very high doses of beta-agonists are commonly selfadministered by patients for prolonged periods prior to hospital presentation with severe asthma. The opportunity exists for clinical review and appropriate medical intervention during this period, which may reduce the risk of a life-threatening attack.

\section{P165 THE RELIABILITY AND PERFORMANCE OF ELECTRONIC MONITORS OF INHALER USE IN A REAL WORLD ASTHMA CLINICAL TRIAL}

${ }^{1} \mathrm{M}$ Patel, ${ }^{2} \mathrm{~J}$ Pilcher, ${ }^{1} \mathrm{D}$ Shaw, ${ }^{3} \mathrm{M}$ Weatherall, ${ }^{2} \mathrm{R}$ Beasley; ${ }^{1}$ Division of Respiratory Medicine, University of Nottingham., Nottingham, UK; ${ }^{2}$ Medical Research Institute of New Zealand, Wellington, New Zealand; ${ }^{3}$ University of Otago, Wellington, New Zealand

\subsection{6/thoraxjnl-2013-204457.316}

Introduction Electronic monitoring is one method to measure the use of inhaled therapy in asthma patients in clinical trials, though the reliability of these devices has been variable. Implementation of trial quality control processes and reporting on the performance of electronic monitors during patient use may help to better understand their utility and limitations. The Smartinhaler Tracker is an electronic monitor for metered dose inhalers (MDIs) that records the date, time and number of actuations to the nearest second.

Objectives In a recently completed 24-week real-world randomised controlled trial of 303 asthma patients at risk of severe exacerbations, Smartinhaler Tracker electronic monitors were used to measure actual use of budesonide/formoterol and salbutamol MDI therapy with two treatment regimens. Our aim is to report on the performance of these monitors, based on the implementation of extensive pre-trial and within-trial validation protocols for their use.

Methods Pre-study use checks involved two actuations of the MDI, with a further two actuations performed at least two hours later. Within-study monitor checks, performed prior to dispensing at follow-up clinic visits, included a computerised check of monitor clock function, actuation accuracy and battery life. Within-study data checks, performed after use of MDIs by participants during the trial, involved computerised checks of monitor clock function prior to data upload.

Results 2678/2728 (98.2\%) monitors passed pre-study use checks; 46/50 monitors failed pre-study checks either because they did not record actuations that were performed, or erroneously recorded extra actuations. 76/2642 (2.9\%) monitors dispensed to participants failed within-study monitor checks; 33/76 monitors failed because the battery was not fully charged. 51/ $2642(1.9 \%)$ monitors failed data upload checks, mostly as a result of fluid immersion during participant use. 93/2642 (3.5\%) monitors were lost or thrown away by participants. Complete data was available from 2498/2642 (94.5\%) of dispensed monitors and 2498/2549 (98.0\%) of returned monitors.

Conclusions The Smartinhaler Tracker is a reliable monitor for measuring MDI use in a real-world asthma clinical trial. Implementation of extensive monitor and data-checking protocols reduces data loss. The use of validated and reliable electronic monitors is the optimal method to assess patterns of inhaled medication use.

\section{P166 EFFECTS OF LOW-VS HIGH-DOSE FLUTICASONE/ FORMOTEROL COMBINATION THERAPY ON AMP CHALLENGE IN ASTHMATIC PATIENTS}

${ }^{1} \mathrm{~F}$ Kanniess, ${ }^{2} \mathrm{Z}$ Diamant, ${ }^{3} \mathrm{M}$ Lomax, ${ }^{3} \mathrm{M}$ Jain; ${ }^{1}$ Practice for Allergy and Family Medicine, Reinfeld, Germany; ${ }^{2}$ Department of Respiratory Medicine and Allergology, Skane University Hospital, Lund, Sweden; ${ }^{3}$ Mundipharma Research Limited, Cambridge, UK

\subsection{6/thoraxjnl-2013-204457.317}

Background The ICS fluticasone propionate (FP) and the LABA formoterol fumarate (FORM) have now been combined in a single aerosol inhaler (FP/FORM; flutiform). The effect of low- (2 puffs $50 / 5 \mu \mathrm{g}$ bid) vs high-dose (2 puffs $250 / 10 \mu \mathrm{g}$ bid) FP/ FORM on airway responsiveness to AMP was compared in an 